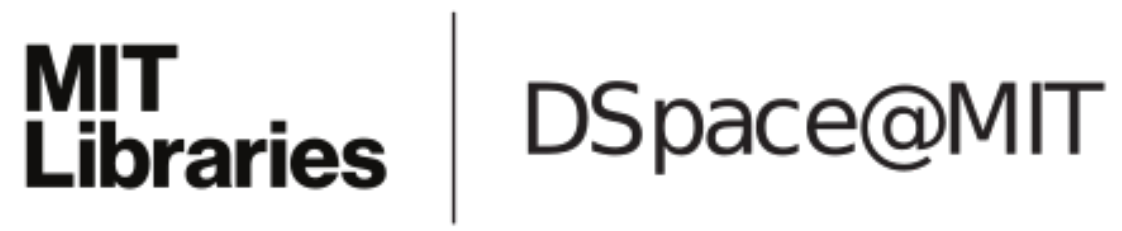

\author{
MIT Open Access Articles
}

The effectiveness of the controlled release of gentamicin from polyelectrolyte multilayers in the treatment of Staphylococcus aureus infection in a rabbit bone model

The MIT Faculty has made this article openly available. Please share how this access benefits you. Your story matters.

Citation: Moskowitz, Joshua S., Michael R. Blaisse, Raymond E. Samuel, Hu-Ping Hsu, Mitchel B. Harris, Scott D. Martin, Jean C. Lee, Myron Spector, and Paula T. Hammond. "The Effectiveness of the Controlled Release of Gentamicin from Polyelectrolyte Multilayers in the Treatment of Staphylococcus Aureus Infection in a Rabbit Bone Model." Biomaterials 31, no. 23 (August 2010): 6019-6030.

As Published: http://dx.doi.org/10.1016/j.biomaterials.2010.04.011

Publisher: Elsevier

Persistent URL: http://hdl.handle.net/1721.1/99390

Version: Author's final manuscript: final author's manuscript post peer review, without publisher's formatting or copy editing

Terms of use: Creative Commons Attribution-Noncommercial-NoDerivatives 


\title{
The Effectiveness of the Controlled Release of Gentamicin from Polyelectrolyte Multilayers in the Treatment of Staphylococcus aureus Infection in a Rabbit Bone Model
}

\author{
Joshua Moskowitz ${ }^{1}$, Michael Blaisse ${ }^{1}$, Raymond Samuel ${ }^{1}$, Hu-Ping Hsu ${ }^{2,3}$, Mitchel \\ Harris $^{3}$, Scott Martin ${ }^{3}$, Jean Lee ${ }^{4}$, Myron Spector 2,3 , and Paula Hammond ${ }^{\text {*', }} 1$ \\ ${ }^{1}$ Department of Chemical Engineering, Massachusetts Institute of Technology, Cambridge, \\ Massachusetts 02139 USA \\ 2 Tissue Engineering Laboratories, VA Boston Healthcare System, Mail Stop 151, 150 S. \\ Huntington Ave., Boston, MA 02130 USA \\ ${ }^{3}$ Department of Orthopedic Surgery, Brigham \& Womens Hospital and Harvard Medical School, \\ 75 Francis St., Boston, MA 02115 USA \\ ${ }^{4}$ Channing Laboratory, Department of Medicine, Brigham \& Women's Hospital and Harvard \\ Medical School, 181 Longwood Avenue., Boston, MA 02115 USA
}

\section{Abstract}

\begin{abstract}
While the infection rate of orthopedic implants is low, the required treatment, which can involve six weeks of antibiotic therapy and two additional surgical operations, is life threatening and expensive, and thus motivates the development of a one-stage re-implantation procedure. Polyelectrolyte multilayers incorporating gentamicin were fabricated using the layer-by-layer deposition process for use as a device coating to deal with an existing bone infection in a direct implant exchange operation. The films eluted about $70 \%$ of their payload in vitro during the first three days and subsequently continued to release drug for more than four additional weeks, reaching a total average release of over $550 \mu \mathrm{g} / \mathrm{cm}^{2}$. The coatings were demonstrated to be bactericidal against Staphylococcus aureus, and degradation products were generally nontoxic towards MC3T3-E1 murine preosteoblasts. Film-coated titanium implants were compared to uncoated implants in an in vivo $S$. aureus bone infection model. After a direct exchange procedure, the antimicrobial-coated devices yielded bone homogenates with a significantly lower degree of infection than uncoated devices at both day four $(\mathrm{p}<0.004)$ and day seven $(\mathrm{p}<0.03)$. This study has demonstrated that a self-assembled ultrathin film coating is capable of effectively treating an experimental bone infection in vivo and lays the foundation for development of a multi-therapeutic film for optimized, synergistic treatment of pain, infection, and osteomyelitis.
\end{abstract}

\footnotetext{
(C) 2010 Elsevier Ltd. All rights reserved.

* To whom correspondence should be addressed. 77 Massachusetts Avenue, Room 66-352, Cambridge, MA, 02139. hammond@mit.edu. Tel.: 617-253-4562. Fax.: 617-258-8992..

Publisher's Disclaimer: This is a PDF file of an unedited manuscript that has been accepted for publication. As a service to our customers we are providing this early version of the manuscript. The manuscript will undergo copyediting, typesetting, and review of the resulting proof before it is published in its final citable form. Please note that during the production process errors may be discovered which could affect the content, and all legal disclaimers that apply to the journal pertain.

Conflict of Interest

The authors confirm that there are no known conflicts of interest associated with this publication.
} 


\section{Introduction}

Osteomyelitis, an inflammatory response to an infecting microorganism that causes bone damage, is a disease that can occur in any bone at any age [1]. One of the major routes of introduction of these microorganisms into the body is postoperative sepsis after orthopedic surgery. The infection rate is on the order of $1 \%$ of the 500,000 joint replacements conducted annually in the United States despite the utilization of aseptic techniques and systemic antibiotics [2]; furthermore, approximately $10 \%$ of all joint replacements are revision operations due to aseptic loosening or infection. The economic and health-related costs to the patient and the health care system are enormous. Specifically, the patient first undergoes an initial surgery during which the original implant is removed, followed by extensive debridement of dead and dying tissues, and implantation of an antibiotic spacer. The patient is subsequently treated with intravenous antibiotics for at least 4-6 weeks. The patient then undergoes a second surgical procedure to implant a new hip prosthesis upon clinical and radiographic determination that infection has been effectively eradicated. This process directly results in a longer hospital stay, prolonged immobility, the possibility of limb shortening, and a total cost estimate that can exceed five times that of the initial surgery [3]. Although relatively effective at eradicating infection, such a two-stage process is a great burden on the patient and can greatly increase the chance of morbidity in weaker or older patients. For these reasons the development of a drug-delivery coating that can eliminate infection and introduce a safe and low-risk direct exchange procedure would be a paradigm shift that will significantly impact the welfare of patients who need revision arthroplasty.

Staphylococcus aureus is the infecting pathogen responsible for about two thirds of chronic osteomyelitis cases [2]. Among the list of antibiotics that are efficacious against most $S$. aureus [4], gentamicin has been selected as the drug of interest in this study, in part due to its broad bactericidal spectrum. Clinically relevant treatment techniques that release gentamicin locally in vivo include bone cement [5], bioactive sol-gel glass [6], injectable polymers [7], polymer coatings [8], and poly(methyl methacrylate) (PMMA) beads [9,10]. For the specific application of orthopedic implant surfaces, the relevant technology platform is a coating. Antibiotic loaded bone cement (ALBC) is commonly used internationally in primary uninfected and in one-stage infected implant surgery. The main drawbacks from using ALBC are: 1) the PMMA reaction is highly exothermic which causes local tissue damage, 2) the un-reacted methyl methacrylate monomer is toxic [11], 3) cemented revisions make future removal much more tedious and difficult to prevent bone damage, and 4) current levels of antibiotic delivery from these cements are insufficient for the direct exchange process, while increased loading malevolently affects mechanical integrity of the cement [12]. Furthermore, bone cements are not able to incorporate multiple therapeutic agents with individualized release rates, and the heat of the polymerization reaction prohibits the incorporation of therapeutic agents such as growth factors due to denaturation. The twostage surgical procedure and several weeks of intravenous antibiotic therapy remains the standard for treatment of staphylococcal implant infection [2], with success rates reported to be over $90 \%[10,13]$.

Our goal was to develop a drug delivery coating that could potentially support a one-stage re-implantation procedure after an infected arthroplasty. To this end, the coating would need to first provide sufficient levels of antibiotics to eradicate an existing bone infection, prevent biofilm formation, and then facilitate new bone formation to ensure intimate contact between the bone and implant surface. Our approach was to develop a biocompatible and biodegradable implant coating constructed from polyelectrolyte multilayers (PEMs) using the layer-by-layer (LbL) technology platform, which is a simple, tunable, economical, scalable, and benign fabrication process for surface coatings. LbL deposition involves the sequential adsorption of complementary species onto an initially functionalized substrate 
[14]. These films have been demonstrated to be conformal to a variety of surfaces [15-17] and inherently have both thickness and dosage control $[18,19]$. PEM films could potentially address the major limitations of ALBC, since they can be designed with biocompatible components without exothermic or other synthetic crosslinking chemistries; for this reason they are of interest for a broad range of orthopedic implant applications including joint and fracture repair. The chief advantage for the use of PEM films is that they can incorporate multiple functional components at very specific regions within the layered film architecture, which can lead to complex and individualized release rates for each type of cargo [20]. Electrostatically assembled PEM films have been developed to release therapeutics such as painkillers [21], anti-inflammatory drugs [22], antibiotics[19], and growth factors [23,24]. With the use of a controllably degradable poly( $\beta$-amino ester) [25], erodible PEM films can deliver therapeutics with individually optimized release profiles to synergistically provide optimal recovery.

The objective of this study focuses on the design and characterization of the antibiotic component of this conceptual multi-drug film. Although the early stages of research directed at the development of LbL coatings for in vivo delivery of therapeutics has just begun [26,27], this study demonstrates proof-of-principle that an LbL coated implant can locally deliver small hydrophilic antibiotics (gentamicin sulfate) and efficaciously treat an S. aureus bone infection in vivo. This study demonstrates an LbL film-based system that improves upon the release durations in earlier reported small-molecule LbL systems [19,21], and is competitive with existing technologies $[6,7,28]$.

\section{Materials and Methods}

\subsection{Materials}

Poly 1 , a poly ( $\beta$-amino ester) depicted in Fig. 1, was synthesized as previously described [29]. Silicon wafers (test grade n-type) were purchased from Silicon Quest (Santa Clara, CA). Medical grade titanium dowels were received from Titanium Industries (Rockaway, NJ). Linear poly(ethyleneimine) (LPEI, 23966-2, $\mathrm{M}_{\mathrm{n}}=25 \mathrm{kDa}$ ) was purchased from Polysciences, Inc. Poly (sodium 4-styrenesulfonate) (PSS, 434574, $\mathrm{M}_{\mathrm{w}}=1 \mathrm{MDa}$ ), Poly(acrylic acid) (PAA, 306215, $\mathrm{M}_{\mathrm{v}}=1.25 \mathrm{MDa}$ ), $3 \mathrm{M}$ concentrated sodium acetate buffer (S7899, pH 5.2), cation-adjusted Mueller Hinton Broth II (CMHB, 90922), and penicillin/ streptomycin solution were purchased from Sigma-Aldrich (St. Louis, MO). Sterile, deionized water and nonradiolabeled gentamicin sulfate (GS) purchased in powder form were from Mediatech, Inc. (Herndon, VA). ${ }^{3} \mathrm{H}-\mathrm{GS}(250 \mu \mathrm{Ci}$ total, $1 \mathrm{mCi} / \mathrm{mL}$ in ethanol, 200 $\mu \mathrm{Ci} / \mathrm{mg}$ ) was obtained from American Radiolabeled Chemicals, Inc. Sodium hyaluronate (or hyaluronic acid (HA), $\mathrm{M}_{\mathrm{n}}=1.7 \mathrm{MDa}$ ) was purchased from Lifecore Biomedical, Inc. (Chaska, MN). Bacto agar and Brucella broth with $20 \%$ glycerol were purchased from BD (Franklin Lakes, NJ). Concentrated castile soap and 70\% v/v isopropyl alcohol pads were kindly donated by Triad Medical (Hartland, WI). All materials and solvents were used as received without further purification.

S. aureus UAMS-1 (ATCC 49230) is a human clinical isolate from a chronic osteomyelitis case [30]. MC3T3-E1 Subclone 4 CRL-2594, a mouse preosteoblasts cell, was obtained from ATCC (Manassas, VA). Alpha minimum essential medium ( $\alpha$-MEM), fetal bovine serum (FBS), trypsin-EDTA, phosphate buffered saline (PBS), Hoechst 33342 dye, and assay kits were purchased from Invitrogen (Carlsbad, CA).

\subsection{Films and In Vitro Experimental Protocols}

2.2.1 Preparation of Polyelectrolyte Solutions-Dipping solutions containing Poly 1 and PAA were prepared at $2 \mathrm{mg} / \mathrm{mL}$ in $100 \mathrm{mM}$ sodium acetate buffer and $\mathrm{pH}$-adjusted to 
5.0 with $1.0 \mathrm{~N}$ sodium hydroxide. GS was prepared at $10 \mathrm{mg} / \mathrm{mL}$ in $100 \mathrm{mM}$ sodium acetate and $\mathrm{pH}$-adjusted to 5.0. LPEI and PSS dipping solutions were prepared in ultra pure water and $\mathrm{pH}$ adjusted to 4.25 and 4.75 respectively. All solutions were prepared with water from a Milli-Q Plus (Bedford, MA) at 18.2 M $\Omega$.

2.2.2 Polyelectrolyte Deposition-In vitro experiments utilized silicon substrates cut to approximately $35 \times 7 \mathrm{~mm}$. Films for in vivo use were built on titanium pegs. All polyelectrolyte LBL thin films were constructed as follows according to the alternate dipping method [14]. Substrates were rinsed with methanol and ultra pure water, dried under nitrogen, and plasma etched in oxygen using a Harrick PDC-32G plasma cleaner at high RF power for 75 seconds. Layer-by-layer thin film deposition was performed using a Carl Zeiss HMS Series Programmable Slide Stainer. A nondegradable bilayer of LPEI/PSS was deposited first by immersion of the plasma treated substrates in LPEI for 30 minutes followed by a single ultra-pure water rinse, and finally in PSS for 30 minutes followed by a single ultra-pure water rinse. The degradable films were deposited on top of this PSSterminated surface. The tetralayer architecture of [Poly 1/Anion/GS/Anion $]_{n}$ was constructed by alternate dipping in a cationic species (i.e. Poly 1 or GS) for 9 minutes followed by a cascade rinse of three $100 \mathrm{mM}$ sodium acetate baths for 10, 20, and 30 seconds, and then into the anionic species (i.e. PAA or HA) for 9 minutes followed by a similar rinse cycle. The process was repeated $n$ times depending on the desired experiment. All polymer solutions and gentamicin solutions were completely replaced every 25 and 50 tetralayers, respectively.

For films used in drug release experiments, the GS solution was spiked with $25 \mu \mathrm{L}{ }^{3} \mathrm{H}-\mathrm{GS}$ per $50 \mathrm{~mL}$ dipping solution yielding a $0.5 \mu \mathrm{Ci} / \mathrm{mL}$ product without significantly changing the concentration of the GS dip bath. No other part of the LbL deposition process was changed.

Films prepared on titanium pegs for the in vivo work were grown to [Poly1/PAA/GS/ $\mathrm{PAA}]_{200}+[\mathrm{Poly} 1 / \mathrm{PAA} / \mathrm{GS}]_{1}$ and allowed to air dry without a final rinse. Additionally, these implants were subjected to a 12-hour cycle of ethylene oxide (EtO) gas for sterilization in an Anprolene AN74i cabinet (Andersen Sterilizers, Inc., Haw River, NC).

\subsubsection{In Vitro Characterization of Film Growth, Drug Release, and Film Erosion}

-Following deposition, all films were allowed to air dry. Films for the growth experiment were scratched with a razor blade and their thickness measured by profilometry at four predetermined locations using a KLA-Tencor P-16 Profilometer. Measurements were performed on three separate films for each tetralayer value, and roughness calculations were performed with the accompanying Profiler Version 7.0 software.

Films used for drug release experiments were stored at $4{ }^{\circ} \mathrm{C}$ until use. Films with ${ }^{3} \mathrm{H}-\mathrm{GS}$ were immersed into $3 \mathrm{~mL}$ of modified simulated body fluid (m-SBF) [31] in a tightly capped Falcon tube maintained at $37^{\circ} \mathrm{C}$. Degradation environments were kept sealed from the ambient to minimize evaporative loss. A $1 \mathrm{~mL}$ sample was extracted from the Falcon tube at predetermined time points and replaced with $1 \mathrm{~mL}$ prewarmed $\mathrm{m}-\mathrm{SBF}$ in a manner so as not to mechanically disturb the hydrated film. The time points were selected with a greater frequency at early times to reflect the kinetics of release. Each extracted sample was mixed with $5 \mathrm{~mL}$ ScintiSafe Plus 50\% (Fisher Scientific, Atlanta, GA) prior to GS quantification. The resulting mixtures were analyzed using a Tricarb Model 2810 TR liquid scintillation counter (Perkin Elmer, Waltham, MA). The raw data in disintegrations per minute (DPM) was converted directly to $\mu \mathrm{g}$ of drug using the DPM value for the dipping solution $(10 \mathrm{mg} /$ $\mathrm{mL}$ ). Total release from the film at the $i$ th timepoint was calculated by the following equation: 


$$
m_{i}=\left(\begin{array}{ll}
C_{i} \times 3 & \mathrm{~mL}
\end{array}\right)+\left(\begin{array}{ll}
1 & \mathrm{~mL}
\end{array}\right) \sum_{j=1}^{i-1} C_{j}
$$

where $m_{i}(\mu \mathrm{g})$ is the the total cumulative mass of GS released from the film at the time of measurement $i, C_{i}(\mu \mathrm{g} / \mathrm{mL}$ ) is the concentration of sample $i$ (which is multiplied by a total of $3 \mathrm{~mL}$ in the Falcon tube), and the summation term adds up the total extensive quantity of gentamicin removed in each of the $i-1$ former aliquots.

Films used for erosion experiments were constructed with the architecture [Poly 1 / PAA / GS / PAA $]_{200}$. As in the release experiments, these films were immersed into $3 \mathrm{~mL}$ of $\mathrm{m}$ SBF in a tightly capped Falcon tube maintained at $37^{\circ} \mathrm{C}$. At each predetermined time point, a minimum of three films were removed from the $\mathrm{m}-\mathrm{SBF}$ and allowed to air dry. The dry state thicknesses were determined via profilometry. Triplicate measurements were made on each film.

2.2.4 S. aureus Antimicrobial Susceptibility Assays-The GS minimum inhibitory concentration was determined according to a previously published microdilution procedure [19] in CMHB with an inoculum of $10^{5} \mathrm{CFU} / \mathrm{mL}$ The microtiter plate was incubated at 37 ${ }^{\circ} \mathrm{C}$ for 16-18 hours, and cell density was read at $600 \mathrm{~nm}$ in a BioTek PowerWave XS Microplate spectrophotometer with accompanying Gen5 program Version 1.00.14. All samples were performed in triplicate. In Kirby-Bauer disk diffusion assays [19], agar plates were inoculated with exponentially growing $S$. aureus in cation-adjusted Mueller Hinton Broth (CMHB) at $10^{8} \mathrm{CFU} / \mathrm{mL}$, and incubated at $37{ }^{\circ} \mathrm{C}$ for $16-18$ hour. The diameter of the inhibition zone was measured in millimeters.

2.2.5 MTT and Cell Viability Assays-MC3T3-E1 cells were grown in $\alpha$-MEM, supplemented with $10 \%$ FBS, and $1 \%$ of antibiotic and antimycotic solution (containing penicillin, streptomycin, and amphotericin $\mathrm{B}$ ) in a humidified incubator $\left(37 \mathrm{C} ; 5 \% \mathrm{CO}_{2}\right.$ in air). Culture medium was replenished every 2-3 days and cells were subcultured when near $100 \%$ confluence with the use of $0.05 \%$ trypsin-EDTA. All cells used in these studies were less than passage number 12 .

Elution buffers for the film degradation studies were prepared by incubating each 100 and 200 tetralayer film in $4.615 \mathrm{~mL}$ growth medium per $\mathrm{cm}^{2}$ of film surface area in tissue culture grade $15 \mathrm{~mL}$ conical tubes at $37^{\circ} \mathrm{C}$ for one week. Cells were seeded at a density of 10,000 cells $/ \mathrm{cm}^{2}$ and incubated at $37{ }^{\circ} \mathrm{C}$ and $5 \% \mathrm{CO}_{2}$ in humidified air for $24-48$ hours prior to exposure to elution buffers in cytotoxicity studies. The culture medium was then changed to medium containing test compounds and incubated with the plated MC3T3-E1 cells for 18 hours prior to evaluation. Parallel MC3T3-E1 cultures were examined by the use of the MTT assay (tetrazolium [3-(4,5-dimethylthiazol-2-yl)-2,5-diphenyltetrazolium bromide] dye, Invitrogen) and the Live/Dead® Viability/Cytotoxicity Kit (Molecular Probes, L-3224) supplemented with Hoechst 33342. The MTT assay was performed according to the manufacturer's specifications.

The Live/Dead® Viability/ Cytotoxicity Kit was used following the manufacturer's recommendations for adherent monolayer cells. The MC3T3-E1 cultures were rinsed with PBS and then examined by fluorescence microscopy with appropriate filter sets for calcein (green cytoplasm of live cells), ethidium homodimer-1 (red nuclei of dead cells), and Hoechst 33342 (blue nuclei of both live and dead cells). Images were analyzed with the use of Image $\mathbf{J}$ software. More details on these assays are provided in the supplementary information. 
2.2.6 Colonization of Implants-Poly(methyl methacrylate) cylinders were fabricated by the MIT Central Machine Shop to be $8.0 \mathrm{~mm}$ long and $2.8 \mathrm{~mm}$ in diameter. The cap of each peg, defined here to be the top $1.0 \mathrm{~mm}$ of the shaft, had a $3.0 \mathrm{~mm}$ diameter. A $1.0 \mathrm{~mm}$ diameter hole was drilled perpendicular to the axis of the rod just under the cap. These rods were suspended from the lid of a glass jar using stainless steel 3-0 gage wire threaded through the $1.0 \mathrm{~mm}$ hole and subjected to EtO gas for sterilization. Under sterile condition $150 \mathrm{~mL}$ CMHB was added to this jar, which completely immersed all suspended rods. $S$. aureus was grown overnight in CMHB (via toothpick inoculation) and added to the jar at a dilution ratio of 1:1000 $(150 \mu \mathrm{L})$. The bacteria were then allowed to surface colonize the acrylic pegs overnight.

The following day, the suspended pegs were subjected to three consecutive $150 \mathrm{~mL}$ sterile water baths, each rinse lasting 60 seconds without agitation. After air drying, the suspended pegs were stored at $-80^{\circ} \mathrm{C}$. All surface colonized pegs were prepared less than one week prior to use.

\subsection{Animals and In Vivo Experimental Protocols}

2.3.1 Approval of In Vivo Procedures-The Committee on Animal Care (CAC) at MIT approved all experiments and animal care procedures. All experiments involving animals were conducted under in a sterile field at a facility certified by the Association for Assessment and Accreditation of Laboratory Animal Care. Sample-size estimation was done using GPower with a significance level of 5\% and a minimum power of $80 \%$ assuming onesided alternate hypothesis (i.e. antibiotic films should decrease infection when compared to the placebo group) [32]. These parameters suggested that at least six rabbits should be used per test group per time point. Thirty-three male New Zealand White rabbits were ultimately enrolled in the present study with weight range of 2.50 to $3.78 \mathrm{~kg}$ (mean and standard deviation, $3.03 \pm 0.32$ ) and were randomized into five test groups: (1) Bone infection model $(n=6),(2)$ four day placebo $(n=6),(3)$ four day treatment $(n=6)$, (4) seven day placebo $(n$ $=7)$, and (5) seven day treatment $(n=8)$.

All rabbits were allowed to acclimate to the facility for a minimum of one week prior to their first operation. They were housed in separate cages in a climate-controlled facility with free access to antibiotic-free food including commercial pellets, hay, and water. Each rabbit was examined by a veterinarian prior to each operation.

2.3.2 Experimental Bone Infection Model-Anesthesia was induced by intramuscular doses of ketamine $(35 \mathrm{mg} / \mathrm{kg}$ ) and xylazine $(5 \mathrm{mg} / \mathrm{kg})$. The rabbits were additionally administered a subcutaneous (SQ) dose of buprenorphine $(0.03 \mathrm{mg} / \mathrm{kg})$ and meloxicam $(0.2$ $\mathrm{mg} / \mathrm{kg}$ ). The left hind leg of each rabbit was shaved from the ankle to the hip and prepared with alternating Betadine scrub and 70\% isopropyl alcohol (IPA). Anesthesia was maintained throughout the operation using $2 \%$ isoflurane gas with oxygen at $1-1.5 \mathrm{~L} / \mathrm{min}$ via an endotracheal tube. Lactated Ringer's solution was administered through a catheter inserted into the cephalic vein, at an initial rate of $10 \mathrm{~mL} / \mathrm{kg}$-hr and then tapered according to observed hemodynamic parameters. A straight medial side incision approximately 3.5-4.0 $\mathrm{cm}$ was centered on the knee joint line. The skin and soft tissue were dissected to the deep investing fascia. A small longitudinal incision was made at the front portion of the superior attachment of the medial collateral ligament (approximately $10 \mathrm{~mm}$ above a branch of popliteal artery) to release the periosteum and expose the medial femoral condylar surface. Using a sterile drill bit, a defect about $8.5 \mathrm{~mm}$ in length was drilled parallel to the axis connecting the medial and lateral condyles. Irrigation was maintained throughout drilling to remove particulate matter. Blood loss was monitored, and animals received three times the estimated loss in Lactated Ringers solution throughout the procedure. After saline joint 
lavage and hemostasis, one $S$. aureus colonized peg was press-fit into the defect. The exposed face of the peg and surgical wire were then cleaned thoroughly with alcohol wipes with the aim of preventing infection unrelated to the bone. The surgical site was closed in layers using 3-0 interrupted sutures for the investing fascia, 3-0 sutures for the superficial fascia and subcutaneous tissue, followed by reapproximation of the skin with a subcuticular stitch using an uninterrupted 4-0 suture. A subcutaneous dose of Lidocaine $(2 \mathrm{mg} / \mathrm{kg})$ was delivered at the surgical site while closing. Each animal was monitored closely until full recovery from anesthesia was observed. Postoperative analgesia was achieved with three subcutaneous doses of buprenorphine (one every 8-10 hours) and two doses of meloxicam (one every 24 hours). Animals were monitored daily for food intake, stool and urine output, and behavior. The residence time allowed for the development of bone infection was four days.

2.3.3 Removal of Colonized Rod and Insertion of a Test Implant-Four days after the initial surgery, six animals were euthanized to determine the bacterial burden in their respective medial femoral condyles. The remaining 27 rabbits were anesthetized and prepared for surgery as described previously. After thorough debridement of the subcutaneous and soft tissue abscess, each acrylic peg was removed and placed into a sterile vial for microbiological analysis (see Sampling Schedule and Analysis of Infection below).

The 27 rabbits were divided into four test groups: four day sterile implants $(n=6)$, four day LbL coated implants $(n=6)$, seven day sterile implants $(n=7)$, and seven day LbL coated implants $(n=8)$. Each test implant was carefully press-fitted into the bone defect. Implants with antibiotic films were dipped in sterile water for less than one second in order to hydrate prior to insertion. Castile soap solution was prepared by adding one $0.3 \mathrm{oz}$. packet to $1.0 \mathrm{~L}$ of sterile water [33]. The subcutaneous and soft tissue areas were thoroughly irrigated using this solution without disturbing the implant or defect. All animals were sutured, dosed with postoperative analgesia, and monitored daily as described above.

2.3.4 Sampling Schedule and Analysis of Infection-In addition to the six animals sacrificed after the bone infection period, six animals from each test group were euthanized at 4 or 7 days after re-implantation. Rabbits were given preanesthesia via intramuscular doses of ketamine $(35 \mathrm{mg} / \mathrm{kg})$ and xylazine $(5 \mathrm{mg} / \mathrm{kg})$. Blood was then drawn from the heart and stored in Vacutainer tubes with anticoagulant (ethylenediaminetetraacetic acid (EDTA) for complete blood counts or heparin for blood cultures) or serum separator elements (for blood chemistry, C-reactive protein, and gentamicin assays). This was followed by euthanasia using an intravenous dose of pentobarbital $(120 \mathrm{mg} / \mathrm{kg})$ injected into the marginal ear vein. Each left knee joint was prepared with Betadine solution, the implant extracted, and stored in a sterile vial. The infected region of each femur was then extracted aseptically by cutting perpendicular to the distal metaphysis axis at about $5.0 \mathrm{~mm}$ from the proximal edge of the drilled defect. All soft tissue was debrided from its surface, and the entire outside surface of the bone was sterilized using $70 \%$ (v/v in water) isopropyl alcohol wipes without disturbing the defect. The bone was then weighed, snap-frozen in liquid nitrogen, and homogenized using a BioPulverizer (BioSpec, Bartlesville, OK). The well-mixed powder was then added to $5 \mathrm{~mL}$ of Brucella broth and stored at $-80^{\circ} \mathrm{C}$ before analysis. Quantitative culturing was initiated after 1 to 4 days of storage. As a control, contralateral femurs of placebo subjects $(n=5)$ were prepared in a similar manner.

Complete blood counts, blood cultures, and microbiology were performed at the microbiology lab within MIT's Division of Comparative Medicine. Blood chemistry was determined by Idexx Laboratories, Inc. (North Grafton, MA), and gentamicin assays were conducted at the Veterinary Medical Center of Cornell University (Ithaca, NY). All explants 
were rolled and streaked on trypticase soy agar plates with 5\% sheep blood (VWR International), and incubated overnight to determine the qualitative extent of surface colonization. The bone homogenates were thawed, vortexed vigorously for 30 seconds, serially diluted, and plated to determine the concentration of bacteria in the homogenate $(\mathrm{CFU} / \mathrm{mL})$. Plates with the largest countable number of bacteria were used in the statistical analyses, and subsequent dilutions with greater than $20 \mathrm{CFU}$ were averaged in with this value. Since the sensitivity of this assay is $10 \mathrm{CFU} / \mathrm{mL}$ (corresponding to $1 \mathrm{CFU}$ on the undiluted plate), a blank undiluted plate was assigned half the value of the experimental uncertainty (i.e. $5 \mathrm{CFU} / \mathrm{mL}$ ). Antibiotic resistance was checked by growing up overnight cultures of bacteria from homogenates, and determining MICs as described above.

2.3.5 Statistical Analysis-Raw data of bacterial populations were not normally distributed and therefore required nonparametric statistical methods. Log-transformed CFU data were analyzed with parametric statistical tests. All statistical analyses were conducted using a Type I error rate of 0.05 , single-tailed testing. Power analyses were performed with the use of G-Power. The Mann-Whitney U-test (nonparametric) was performed in StatView (SAS Institute Inc.). F-tests for variances and subsequent t-tests for means were computed using the Analysis Toolpack in Microsoft Excel. Statistical power greater than 0.80 and pvalues smaller than 0.05 were considered to be significant.

\section{Results and Discussion}

\subsection{Designing an Antimicrobial Film Using Electrostatic Layer-by-Layer Self-Assembly}

When designing layer-by-layer systems for the release of antibiotics in a physiological environment, there are three basic material requirements that must be addressed with respect to film components: (1) incorporation of a therapeutic, (2) a mechanism of release, and (3) any other materials necessary to permit stable layer-by-layer film growth without compromising biocompatibility. The antibiotic gentamicin sulfate is a small, water-soluble aminoglycoside with five amine groups that are protonated at low $\mathrm{pH}$ to a maximum charge of +5.0 (Fig. 2, left), and thus easily incorporated into thin film systems that rely on electrostatic interactions [19]. Additionally, gentamicin has a minimum inhibitory concentration of $0.156 \mu \mathrm{g} / \mathrm{mL}$ against $S$. aureus UAMS-1 (Fig. 2, right) which is consistent with literature values for other strains of $S$. aureus [4]. Because gentamicin is nephrotoxic and ototoxic at elevated systemic levels, the low concentration necessary for local delivery via LbL systems is advantageous.

To enable hydrolytic breakdown in the physiological environment $\left(37^{\circ} \mathrm{C}, \mathrm{pH} 7.4\right)$, we used a class of polycations known as poly( $\beta$-amino esters), which are easily synthesized by stepwise conjugation of bis amine monomers to diacrylate esters [29]. These polymers undergo hydrolytic cleavage of the ester bonds, and have been incorporated successfully into multilayer films for controlled release [25,34]. We chose to work specifically with the polymer species shown in Fig. 1, which we define in this paper as Poly 1, due to its relatively slow degradation rate and high charge density [25]. The amines present along the backbone of Poly 1 are protonated at neutral to low $\mathrm{pH}$, yielding the positive charge necessary for electrostatic LbL systems. Neither Poly 1 nor its small molecule degradation products were found to be cytotoxic toward fibroblastic NIH3T3 cells [29], monkey kidney Cos-7 cells [35], or even murine pre-osteoblast MC3T3 cells [19].

Both of the functional components-gentamicin and Poly 1 -are positively charged under our dipping conditions ( $\mathrm{pH}$ 5.0). Therefore, an anionic component is required to allow for the stable growth of the LbL film. The biopolymer HA had previously been employed in our lab for this purpose [19]; however, PAA had already been used in its bulk form as both a gentamicin delivery vehicle in an in vivo osteomyelitis model [36] and an orthopedic 
implant coating to enhance the biocompatibility of titanium-based surfaces [37]. Unlike HA, PAA can act as a diffusion barrier within multilayer films [38], and has a much larger glass transition temperature $\left(\mathrm{T}_{\mathrm{g}}\right)\left(106{ }^{\circ} \mathrm{C}\right.$ compared to about $\left.14{ }^{\circ} \mathrm{C}\right)$, thereby resulting in mechanically more rigid films that release the relatively diffusive gentamicin over a longer timescale. PAA was chosen for our studies after an initial set of GS release experiments comparing it to HA using the architecture [Poly1 / Anion / GS / Anion $]_{50}$; the polyanions are compared in Fig. 3. The 1.25 MDa PAA films $(\mathrm{n}=4)$ incorporated about 7.5 times more GS than the 1.7 MDa HA films $(n=8)$. This could be partly due to the difference in charge density along each respective polymer backbone (i.e. difference in total charges per mass of repeat unit for each polymer). Given identical polymer concentrations by mass in the fully charged state, the PAA would have approximately 5.26 times more charge than the HA. This results in comparatively less interaction and bonding of HA with GS in the multilayer films.

The screening experiments also sought to determine the benefit of using 1.25 MDa PAA over $100 \mathrm{kDa}$ PAA. It was found that the high molecular weight PAA $(\mathrm{n}=4)$ did indeed incorporate greater amounts of drug than the low MW counterpart $(n=6)$, but not by a significant margin. Subsequent experiments utilized the 1.25 MDa PAA.

\subsection{Growth Curve of [Poly 1/PAA/GS/PAA $]_{n}$}

One of the major advantages to LbL systems is that the thickness of the films can be tuned according to the total number of layers deposited. Since drug is cyclically incorporated throughout the growth process as one of the structural components, the total drug loading scales with thickness and is therefore also tunable (but not independently). The [Poly $1 /$ PAA/GS/PAA $]_{n}$ architecture resulted in stable and reproducible film growth up to 200 tetralayers (Fig. 4).

Several studies have sought to explain differences in observed growth behavior of $\mathrm{LbL}$ systems [39-44]. The simplest systems grow solely in linear fashion as polyelectrolytes in solution interact only with the oppositely charged outermost layer of the growing film. Bilayer thicknesses during basic linear growth range from the nanometer scale to tens of nanometers. Exponential growth is generally observed when there is at least one diffusive component that absorbs into the bulk of the growing multilayer film, and each subsequent deposition cycle leads to increased increments of material absorbed. Based on our data, for which the average bilayer thickness is on the order of $150 \mathrm{~nm}$, we believe that our films begin building in the exponential regime due to the diffusive nature of gentamicin (477 Da). After the film is grown beyond a threshold thickness-which takes place before the 25 tetralayer data point - there is a transition from the exponential regime to a secondary linear regime. This occurs when the film becomes so thick that the time provided for diffusion (i.e. 9 minute dip cycles) is less than the time necessary for the gentamicin to access the entire thickness of the film via diffusion. As a result, this diffusion-limited growth curve appears despite the presence of a diffusive component, but exhibits much larger bilayer thicknesses. Details of this transition and how to control its onset are described elsewhere [42,43]. One implication of this behavior is that we expect the concentration of gentamicin to be greatest in the diffusion zone of the film (i.e. the outermost regions), which therefore contributes to an initial burst-release behavior of drug followed by a more controlled linear release.

The surface roughness increases with the total number of deposited tetralayers until about $\mathrm{n}$ $=50$, after which it remains relatively constant $(8.6 \pm 1.6 \mu \mathrm{m})$ as observed both with profilometry and scanning electron microscopy (data not shown). The unusually high values of roughness in the current system are likely a result of significant diffusion of PAA, which is a weak polyanion with a solution dissociation constant $(\mathrm{pKa} \approx 6.5)[45]$ that lies above our deposition condition $(\mathrm{pH}=5.0)$, and the small positively charged molecule gentamicin. The combination of interdiffusion and some small amount of film dissolution during the 
assembly period is a likely cause of some diffusive loss of material from the film and roughness introduced during the deposition process.

\subsection{In Vitro Kinetics of Gentamicin Release from Films}

We aimed to design films that could treat an existing bone infection, and to this end, an ideal release profile would contain an initial burst release of drug over the first few days to sterilize the infected implant site, followed by a prolonged zero-order release that would effectively prevent any surviving bacteria from multiplying and re-colonizing the surface of the implant. The extended release should last weeks to allow the patient's immune system to resolve the infection. Therefore, films were built with $[\text { Poly 1/PAA/GS/PAA }]_{200}+[$ Poly $1 /$ $\mathrm{PAA} / \mathrm{GS}]_{1}$ for a total of 803 deposited individual layers (on top of the nondegradable baselayers). The final adsorption step was of gentamicin, which allowed excess GS to permeate and load into the film during this final deposition step without a final rinse. This provided a means of incorporating excess unbound drug available for immediate diffusive release upon immersion into a physiological environment (approximately $20 \%$ increase in total drug load from the 200 tetralayer films), which would be followed by gradual surfaceerosion based degradation of the remaining drug-containing film.

The films $(n=3)$ released approximately two thirds of their total load within the first three days in m-SBF maintained at $37{ }^{\circ} \mathrm{C}$ (Fig. 5A). This aqueous buffer closely models the conditions of human blood plasma without the biological components [31]. We believe that there are three major factors contributing to this burst release: 1) excess unbound drug diffuses out of the film, 2) m-SBF has a pH of 7.4 which causes some de-protonation of the amines that were originally loaded into the film at $\mathrm{pH} 5.0$, thereby reducing the charge state of GS to approximately +3.5 [46] and facilitating the release of loosely interacting molecules, and 3) the salts present in the m-SBF diffuse into the film and compete for the same ionic sites as the GS, which further enhances drug release. Since the films exhibit super-linear growth behavior with gentamicin as one of the key diffusing species, it is anticipated there is a GS-rich diffusion zone at the film-solution interface $[42,43]$. It is likely that the rapid loss of GS from this region of the film may trigger subsequent film instability and deconstruction, which leads to more rapid release than would be expected from hydrolysis of Poly 1 alone. This is, in part, supported by assessment of film erosion data (Fig. 5A). Films were grown to 200 tetralayers and immersed in m-SBF. They were removed at pre-determined time points and their dry state thicknesses determined by profilometry on at least triplicate samples with triplicate measurements per sample. The film thickness decreased rapidly during the burst release phase of drug elution and was followed by a relatively linear erosion behavior that is consistent with zero-order release. The effects of the three putative mechanisms noted above are expected to play an important role primarily during burst release phase, as the zero-order release phase pertains to strongly bound drug and the stabilized film, which erodes due to hydrolytic degradation of the polycation.

Beyond day three, the films continue to release drug in a relatively linear fashion until their expiration at 5.5 weeks (Fig. 5B). This compares favorably against the rapid release architectures based on hyaluronic acid [19]. The average total loading of these films exceeded $550 \mu \mathrm{g} / \mathrm{cm}^{2}$. From days 3 to 13 , the films released over $11 \mu \mathrm{g} / \mathrm{cm}^{2} /$ day, and during the subsequent four weeks, the films released over $4 \mu \mathrm{g} / \mathrm{cm}^{2} /$ day. The diffusivity of gentamicin in different environments including collagen, agar, and mucus, has been reported to be on the order of $10^{-6} \mathrm{~cm}^{2} / \mathrm{s}[47,48]$, which would translate to a local linear velocity that is less than $1 \mathrm{~cm} / \mathrm{hr}$. If this is consistent with the behavior in the local joint, then the concentration of drug would exceed the MIC of gentamicin against $S$. aureus for over five straight weeks from the initial implant date. It is expected that when water permeation into the LbL films is significantly faster than the rate of hydrolytic degradation of Poly 1 , then 
the films would degrade in a bulk erosion-type manner since the entire film could degrade simultaneously; however, when water diffusion is significantly slower than hydrolysis of Poly 1 , then we expect surface erosion since the topmost layer would degrade and dissociate first. The linear release phase observed in our films is indicative of a controlled, surfaceerosion based release that is consistent with the observed zero-order erosion behavior in Fig. 5A. Theoretically, if the drug concentration throughout the film thickness in this region of the film is constant, and no gradients develop within the film during release, then the normalized film thickness and released drug amounts should total to 100\%; as shown in Fig. $5 \mathrm{~A}$, these data are consistent with the model of linear scaled release.

Any implant or device inserted into the body of a human or animal must be sterilized in order to prevent the possibility of subsequent infection. EtO gas treatment is a room temperature process that subjects the film to a highly effective killing agent without chemically changing the film components, and therefore film performance is preserved. Our films were subjected to a 12 hour EtO cycle and subsequently stored for at least 24 hours to allow any residual gas to dissipate from the film. After comparing sterile and non-sterile films in triplicate, EtO gas appeared to have virtually no effect on release kinetics, and did not significantly alter the total loading of gentamicin in our LbL films $(\mathrm{p}=0.48)$ (Fig. 5B).

\subsection{In Vitro Activity of Films against Staphylococcus Aureus via Kirby-Bauer Assays}

One of the deliverables of this study was to confirm the killing efficacy of the LbL constructs against $S$. aureus in an in vitro assay and further ensure that the EtO sterilization procedure does not change release kinetics. Kirby-Bauer disk diffusion assays provide both qualitative information and a quantitative estimate of the amount of gentamicin that has diffused through agar by measuring the zone of inhibition (ZOI). [Poly 1/PAA/GS/PAA] 200 $+[\text { Poly 1/PAA/GS }]_{1}$ films were grown on identical titanium rods with dimensions similar to those used in the in vivo experiment. One of the rods was subjected to EtO sterilization while the other was left unsterilized. Each rod was pressed into a separate agar plate and BD Sensi-Disc standards with $10 \mu \mathrm{g}$ of gentamicin were used to ensure that the ZOIs could be accurately compared. The ZOI was virtually unchanged by the 12 hour EtO cycle, which additionally supports the fact that EtO is an effective way to sterilize our LbL system without altering film loading or release kinetics. Furthermore, the Kirby-Bauer diameter, which is the diameter of the ZOI minus the linear dimension of the eluting agent (e.g. the rod), was approximately $22.8 \mathrm{~mm}$ in comparison to the $18.1 \mathrm{~mm}$ generated by the SensiDisc standard (Fig. 6); in vitro diameters that exceed $15 \mathrm{~mm}$ are generally regarded as a good predictor of effective treatment against $S$. aureus [49]. Under the simplified assumption that the concentration of drug in an eluting agent is proportional to the ZOI, and recalling the release curve data of these films in aqueous $\mathrm{m}-\mathrm{SBF}$, we expected a much larger ZOI relative to that of the Sensi-Disk than what was observed. Therefore, it is possible that the gelatinous nature of the agar inhibits not only the effects of burst release resulting from $\mathrm{pH}$ change and salt competition, but also the rate at which water can hydrolytically degrade our films to yield release of all the encapsulated gentamicin.

\subsection{Cytotoxicity toward MC3T3 Cells}

MTT assays were run to determine the cytotoxic effect of our films against murine MC3T3E1 cells, subclone 4 with high osteoblast differentiation and mineralization activity. We designed this experiment to mimic an overcompensated situation where films were eluted at $37^{\circ} \mathrm{C}$ for one week ( $\approx 70 \%$ of cargo) into a small buffer reservoir forming a more concentrated test sample relative to what would be experienced in vivo. We repeated this experiment in triplicate for films of 100 and 200 tetralayers, and compared the results to a negative control of untainted $\alpha$-MEM buffer. Since the in vivo environment is both a larger reservoir and an open system, the concentrations of degraded material achieved in this in 
vitro experiment should exceed those that would be experienced by the same films in vivo barring any local gradients at the film surface that would exist in any antibiotic film-eluting system.

There was no statistical difference in metabolic activity of the cells between the control and 100 tetralayer elution buffers in this assay (Fig. 7A). However, the 200 tetralayer elution buffers did cause a significant reduction in the metabolic activity of the cells. Upon further investigation via the Live/Dead $®$ assay and direct imaging with fluorescence microscopy, it was confirmed that the 100 tetralayer films are benign towards the MC3T3-E1 cell line and present no apparent biocompatibility issues (Fig. 7B). In contrast, the 200 tetralayer elution buffer (Fig. 7C) causes a clear reduction in cell viability as a result of the increased concentration of eluted material. Dead cell percentages were calculated for each test group from cell counts of images (Fig. 7D). The discrepancy between the reduced metabolic activity $(\sim, 70 \%$ reduction) and the cell viability $(\sim, 25 \%$ reduction) suggests that the metabolic activity per cell is also reduced. These cytotoxic effects must be a result of either Poly 1 (or degradation products), GS, PAA, or complexes. Past studies have determined the biocompatibility of Poly 1 towards a variety of cell lines [19,29,35], and PAA is an FDAapproved, biocompatible material that has been studied for use as a surface coating to enhance the biocompatibility of titanium implants [37]. It is well-known that GS is nephrotoxic and ototoxic at elevated systemic concentrations, which reinforces one of the main benefits of localized drug delivery systems. A 200 tetralayer film contains roughly 600 $\mu \mathrm{g} / \mathrm{cm}^{2}$ of GS, which after one week would have eluted about $450 \mu \mathrm{g} / \mathrm{cm}^{2}$ of drug (i.e. $70 \%$ ) into the $4.5 \mathrm{~mL} / \mathrm{cm}^{2}$ of buffer, yielding a $100 \mu \mathrm{g} / \mathrm{mL}$ solution of GS. Isefuku et al. determined that GS concentrations above $100 \mu \mathrm{g} / \mathrm{mL}$ caused reduction in osteoblast alkaline phosphatase activity [50]. At higher concentrations of GS, osteoblast cell proliferation was affected. We believe that the GS - whether complexed or not-is likely the culprit for the onset of the observed cytotoxic effects of the 200 tetralayer elution buffers.

\subsection{Clinical Evaluation in New Zealand White Rabbits Pretreatment Period: Bone Infection Model}

Before studies can be conducted to find new ways to treat osteomyelitis, one must be able to establish a reproducible bone infection in vivo. S. aureus can be introduced to the host via direct inoculum or by implantation of a colonized foreign body. This study uses the latter approach as it is more directly relevant to the application of a contaminated implant during primary arthroplasty, and it provides an implant surface onto which bacteria may form a biofilm. Sessile bacteria embedded within a biofilm are notoriously more resistant to antimicrobial therapy than planktonic bacteria cultivated in the same medium.

Our bone infection model was designed to reproducibly simulate a primary surgery that introduces implant-related osteomyelitis. Although less frequently employed than direct bacterial inocululation, foreign bodies have been used in past in vivo studies to increase the rate of infection due to biofilm formation on the implant surface and render the bacteria more resistant to eradication by host immune defenses [51]. Infection by S. aureus was successfully contained near the defect site since cultures of the blood from all rabbits and contra-lateral femurs from five rabbits were sterile. After the pretreatment period each surgical site was reopened, and the contaminated PMMA rod extracted and plated. These cultures indicated the presence of $S$. aureus exclusively as the infecting agent. The plates provide a qualitative metric on the extent of surface colonization of the explanted rods (Supplementary Fig. 1), and suggest that there was ongoing infection within the defect site.

The body weights of infected rabbits decreased significantly throughout the bone infection period. The rabbits lost an average of $9.1 \pm 3.3 \%$ of their weight (with a maximum of $15.4 \%$ and minimum of $3.0 \%, \mathrm{n}=33$ ) over the four-day period. Although all thirty-three rabbits 
underwent the pretreatment procedure, six rabbits were euthanized without re-implantation for quantitative analysis of bone cultures and blood chemistry analysis. After determination of $\mathrm{CFU} / \mathrm{mL}$ in the bone homogenates by serial dilution, the total CFU present within the extracted bone was back-calculated using the bone concentration in the homogenate $(\mathrm{g} / \mathrm{mL})$ and total bone extracted from the rabbit $(\mathrm{g})$. The $\log _{10} \mathrm{CFU}$ per defect site for these six rabbits was $6.36 \pm 0.94$ (mean \pm standard deviation), which confirms a reproducible and highly infected defect site.

Complete blood counts $(n=9)$ and blood chemistry $(n=6)$ data were generated for healthy rabbits prior to the pretreatment period. These values were compared to those of the infected animals after the pretreatment period ( $\mathrm{n}=6$ for both CBC and blood chemistry). Twelve metrics proved to be statistically significant in differentiating these two groups (Supplementary Table 1). The strongest data in support of bacterial infection were the total CFU counts, weight loss, the positive explant cultures, and leukocyte counts. The increased leukocyte concentration ( $p$-value $<0.01$ ) is generally related to the presence of infecting microorganisms. Additionally, there was an increase in the proportion of neutrophilic leukocytes in the infected rabbits (50\% vs. $30 \%$ in healthy rabbits), which is consistent with acute bacterial infection.

In this investigation, the objective of the bone infection model was to evaluate proof-ofprinciple efficacy data from LbL release films in an in vivo environment, while maintaining relevance to the application of conducting a one-stage direct exchange procedure to replace an infected prosthesis. There are two major limitations of this model. First, our method of introduction of bacteria was non-quantitative. The PMMA rods were allowed to incubate overnight in a S. aureus broth culture with the aim of establishing a substantial biofilm on the implant surface. As a result, the number of CFU in the bacterial challenge to the defect is unknown. We elected to use the colonized implant because it simulates the case of a contaminated primary arthroplasty; also, introduction of a bacterial suspension into the bone defect led to unacceptable levels of sepsis in our pilot studies. Regardless, the bacterial burden at the end of the four day bone infection period prior to treatment is more important than the initial inoculum. Ultimately, the amount of infection achieved following the four day incubation period was quantitated, as discussed above. The second limitation to our model was that subcutaneous soft tissue infection local to the defect site was difficult to control. There was no treatment for infection unrelated to the bone in this study, leading to some unnecessary infectious burden on the animals, ultimately resulting in weight loss and occasional sacrifice before later time points ( $>1$ week) could be studied. The presence of infection external to the bone obviates meaningful comparisons of blood data between the placebo and treatment groups since the treatment is restricted to the bone defect site. Soft tissues were meticulously cleaned and removed from the bone prior to homogenization and did not significantly affect the bone cultures, which are the primary metric of efficacy in our proof-of-principle study. Future development of the model may include sealing off the implants using bone wax.

\subsection{Clinical Evaluation in New Zealand White Rabbits Posttreatment Period: Direct Exchange and Follow-Up}

In order to prevent any possibility of shrouding the effect of the antibiotic LbL film, we intentionally chose to omit two major relevant clinical procedures that would otherwise be used in a human operation: the use of systemic antibiotics and debridement of the bone defect. After the four day pretreatment period, twenty-seven rabbits underwent a direct exchange operation in which either an uncoated titanium implant (placebo, $\mathrm{n}=13$ ) or filmcoated titanium implant (treatment, $\mathrm{n}=14$ ) replaced the incumbent, colonized PMMA rod. There was no other tampering of the highly colonized defect site. Soft tissue complications were not considered within the scope of this study. Consequently, to reduce unnecessary 
burden on the animals, all abscesses were drained of pus and both infected and necrotic soft tissues external to the defect site were thoroughly extracted before a final wash using castile soap solution. These procedures were conducted with an implant in place and care was taken to ensure that the implant site was not disturbed.

Six animals from each of the placebo and treatment test groups were euthanized four days after direct exchange. Films were not completely eroded at this time point. Five of six animals (83\%) in the placebo group had $>200 \mathrm{CFU}$ of S. aureus on their explanted titanium device compared to zero of six animals in the treatment group (maximum of $22 \mathrm{CFU}$ ). Total colonization of devices in the treatment group was substantially lower than that of the placebo group (Fig. 8A). Total CFU counts in the bone homogenates confirmed gross infection in six of six animals from the placebo test group, whereas three of six animals in the treatment group yielded sterile bone cultures (Fig. 8C). A Mann-Whitney U-test comparing the underlying distributions of raw CFU data at the four day time point indicated that the reduction in CFU counts due to the antimicrobial LbL films was statistically significant $(\mathrm{p}<0.004)$ when compared to those of the placebo test group. This was further verified by analyzing the log-transformed data via an F-test for variances $(p>0.15)$ and subsequent $\mathrm{t}$-test assuming equal variances $\left(\mathrm{p}<1 \times 10^{-8}\right)$. At four days after direct exchange, the antibiotic films decreased the average bacteria burden by an average of almost five orders of magnitude.

One week after direct implant exchange, the remaining fifteen animals were euthanized (placebo $\mathrm{n}=7$ and treatment $\mathrm{n}=8$ ). Films were still not completely eroded at this time point. Five out of seven animals $(71 \%)$ in the placebo group had S. aureus colonization that exceeded $200 \mathrm{CFU}$ on their explanted devices compared to zero out of eight animals in the treatment group (maximum was $190 \mathrm{CFU}$ ). A qualitative assessment of the total surface colonization of the explanted devices on day seven indicated that the LbL coatings continued to prevent re-colonization of the implant surface when compared to the placebo films (Fig. 8B). This result is favorable since infectious complications become much more robust and hostile if biofilm formation occurs on the implant surface. While all animals in the placebo test group had sizeable CFU counts, two of eight animals (25\%) in the treatment group did not have detectable quantities of bacteria. The Mann-Whitney U-test $(\mathrm{p}<0.03)$, Ftest $(\mathrm{p}<0.05)$, and subsequent $\mathrm{t}$-test assuming unequal variances $(\mathrm{p}<0.02)$ confirmed that the two order of magnitude reduction in CFU counts was statistically significant (Fig. 8C). There was no significant decrease in CFU count from day four to day seven within the placebo group. In this case, the staphylococcal infection is only controlled by the host immune system since no antimicrobial agents were introduced during the direct exchange procedure. The increase in variance and magnitude of infection for the treatment group between days four and seven, however, was significant (F-test, $\mathrm{p}<0.005$ and t-test, $\mathrm{p}<$ $0.01)$. The increase in variance can be attributed to the fact that unsaturated bacteria populations tend to have non-steady state behavior. Specifically, they will either continue to propagate until all available resources are utilized, or be killed off in the presence of bactericidal agents until the population is eradicated. This behavior is evident in MIC assays since the wells of the microtiter culture plate are either turbid or clear, depending on the antibiotic concentration used in each well. In the case of the rabbits, six of the eight subjects in the treatment group were not completely eradicated of bacteria at day seven. If the rate of bacteria propagation exceeds the rate of treatment, then the animal would not be cured and eventually endure bacterial infections similar to those of the placebo group. Since the in vitro elution profile of these LbL films yields a monotonically decreasing drug release rate, and since the bolus release phase has already passed, it is unclear whether the release rate of the remaining drug in the uneroded film would be sufficient to clear these six animals of infection at later time points. Regardless, the final CFU counts at equilibrium would be either near zero or on the order of $10^{6}$ (according to our infection model), which is the 
reason for the observed difference in variances. The increase in magnitude of infection at day seven could have further been a result of a potential re-seeding issue due to the growing adjacent subcutaneous infection. Antibiotic resistance of the bacteria from the seven day treatment group has been ruled out after testing their susceptibility to gentamicin (Supplementary Fig. 2).

Both serum and the supernatants of centrifuged bone homogenates were sent to Cornell University's College of Veterinary Medicine for gentamicin quantification by fluorescence polarization immunoassay (FPIA). As expected, there was no detectable amount of gentamicin in any serum or placebo homogenate sample that was analyzed in this study (detection limit $=0.27 \mu \mathrm{g} / \mathrm{mL}$ ). The four day treatment group had $60.5 \pm 34.9 \mu \mathrm{g}$ GS (maximum $83.2 \mu \mathrm{g}$, minimum $18.4 \mu \mathrm{g}, \mathrm{n}=6$ ) remaining in the condylar region of the femur after removal of the coated device. The seven day treatment group had one animal with significantly higher GS quantity than the other seven animals (i.e. $259.1 \mu \mathrm{g}$ ), which was likely due to residual film that detached from the titanium implant surface upon extraction and therefore remained in the defect site after implant removal. After omitting this outlier data point from further analysis, the seven day treatment group had $23.4 \pm 15.8 \mu \mathrm{g}$ GS (maximum $57.9 \mu \mathrm{g}$, minimum $12.1 \mu \mathrm{g}, \mathrm{n}=7$ ). Since it was not clear whether the GS would be efficiently flushed away from the surgical site on the timescale of one week, a two-tailed $\mathrm{t}$-test was conducted to compare the means of the four- and seven-day treatment groups ( $\mathrm{p}<$ 0.05 ). The significant decrease in GS quantity at seven days in comparison to four days implies that the rate of uptake of drug by the body exceeded the rate of elution by the LbL coatings over these three days. This result is important in light of the in vitro cytotoxicity assays, which determined that without sufficiently fast pharmacokinetics, gentamicin concentrations could potentially build up and reach locally toxic levels. As expected, this is not the case in the in vivo study; however, the homogenized GS quantities at both four and seven days after implantation do continue to exceed the MIC of GS against $S$. aureus (i.e. $0.1 \mu \mathrm{g} / \mathrm{mL}$ ) (assuming that the condylar region of a single rabbit femur has a volume on the order of milliliters).

Following the results of the in vitro release profile, we expected to see a reduction in bacterial bone counts between treatment and placebo groups by day four since this corresponds to the conclusion of the burst release phase. Had the staphylococci been completely eradicated, the day seven comparison should have expanded these differences. However, under the condition of incomplete eradication (the case for some of the treated animals in this study), the bacteria were able to re-propagate under the diminishing antibiotic release rate of the LbL films and re-establish gross infection. Our bone infection model was designed to present a bacterial challenge with a $100 \%$ infection rate, and address this infection solely with the use of our antibiotic films. In an actual revision operation in a human with an infected implant site, the role of systemic antibiotics and defect management are tremendously important. The current standard of care for patients undergoing orthopedic surgery requires receipt of a preoperative systemic dose of a cephalosporin antibiotic, which is capable of diffusing out of capillary beds to assist with infection control [52]. Additionally it is well known that adequate surgical debridement and cleaning of a previously infected defect site before insertion of a new implant are the most important practices in infection control. In practice, the irrigation procedure involves a high pressure rinse of soap or antibiotic solution [33] to help forcibly remove any sessile infectious microorganisms. Future investigations of follow-up times greater than one week may benefit from co-delivery of systemic cefazolin, and cleaning of the defect site (e.g. with hydrogen-peroxide and povidone-iodine solutions) while being careful to account for proper controls. Under this revised procedure, the bacterial burden placed on the LbL films would be reduced and the seven day treatment group may have yielded a greater degree of eradication. 
The results presented here have achieved the primary aim of this study, which was to generate proof-of-principle data that support a reasonable measure of efficacy of an antibiotic eluting LbL system in vivo. There are a few key limitations to the present study. Histological and micro-computer tomographical analyses, which would yield additional information on bone healing and local host responses to the bacterial infection and antibiotic coatings, were not conducted, and dose response was not examined in this study. Although they were deemed beyond the scope of this study, these studies will be necessary when assessing long-term viability of this approach. As noted above, our bacterial challenge was high and our one-stage re-implantation did not involve any systemic antibiotics or cleaning of the infected bone defect as would be done in an actual clinical case. Nonetheless, we were able to demonstrate remediation of bone infection over the course of days. Future work that addresses multi-component release or aims to treat an existing infection in a larger animal model with human sized implants can use an infection model that employs a smaller inoculum challenge or supplements the treatment with systemic antibiotics and proper debridement, to better represent a more realistic clinical situation.

\section{Conclusions}

In summary, thin films with antibiotic functionality were constructed in layer-by-layer fashion by alternating the deposition of a hydrolytically degradable poly( $\beta$-amino ester), biocompatible poly(acrylic acid), and the therapeutic gentamicin that required no premodification. These polyelectrolyte coatings released their loaded drug through both diffusion and film erosion when placed into aqueous environment yielding a burst-release of cargo over the first several days, followed by weeks of continuous zero-order release via hydrolytic erosion. The films were effective in inhibiting the in vitro growth of $S$. aureus and benign to MC3T3-E1 murine pre-osteoblast cells except under concentrations that are consistent with toxic levels of gentamicin. In vivo, the polyelectrolyte-coated titanium implants significantly decreased the viable bacteria count compared to the uncoated placebo in a simulated one-stage re-implantation model to treat implant-related $S$. aureus infection in New Zealand White rabbits. Furthermore, the antimicrobial films inhibited bacterial growth on the implant surface when compared to the uncoated placebo. Overall, this study reports the design and implementation of LbL coatings to effectively treat a gross $S$. aureus infection in vivo via local delivery of common clinical antibiotics in a simulated direct exchange procedure. These results are an important step forward in the broader effort of developing complex release architectures that combine antibiotics with growth factors and analgesics for optimized, multi-drug release.

\section{Supplementary Material}

Refer to Web version on PubMed Central for supplementary material.

\section{Acknowledgments}

This work was financially supported by the National Institutes of Health, National Institute of Aging (5R01AG029601-03). We thank Catrina Wong, Alison Hayward, DVM, and Alison Darby, DVM (animal support staff, Division of Comparative Medicine, MIT) for care, handling, and anesthesia of animals, Ellen Buckley and Nikki Lew (microbiologists, Division of Comparative Medicine, MIT) for blood analyses, cultures, and biofilm studies, Wayne Schwark, DVM, PhD (Professor of Pharmacology, Department of Molecular Medicine, Cornell University), for gentamicin assays, and Helen Chuang, PhD (Research Scientist, ChromoLogic, Pasadena, CA), for consultation on in vitro film studies. The authors greatly appreciate the use of equipment available at the Institute for Soldier Nanotechnology (ISN), as well as the Robert Langer Laboratory for scintillation counting, the Center for Materials Science and Engineering for profilometry, and the Division of Comparative Medicine's animal facilities for all in vivo work. 


\section{References}

1. Lew DP, Waldvogel FA. Osteomyelitis. Lancet 2004;364(9431):369-379. [PubMed: 15276398]

2. Soundrapandian C, Datta S, Sa B. Drug-eluting implants for osteomyelitis. Crit Rev Ther Drug 2007;24(6):493-545.

3. Fisman DN, Reilly DT, Karchmer AW, Goldie SJ. Clinical effectiveness and cost-effectiveness of 2 management strategies for infected total hip arthroplasty in the elderly. Clin Infect Dis 2001;32(3): 419-430. [PubMed: 11170950]

4. Andrews JM. Determination of minimum inhibitory concentrations. J Antimicrob Chemoth 2001;48:5-16.

5. Buchholz HW, Elson RA, Heinert K. Antibiotic-loaded acrylic cement - Current concepts. Clin Orthop Relat R 1984;(190):96-108.

6. Meseguer-Olmo L, Ros-Nicolas MJ, Clavel-Sainz M, Vicente-Ortega V, Alcaraz-Banos M, LaxPerez A, et al. Biocompatibility and in vivo gentamicin release from bioactive sol-gel glass implants. J Biomed Mater Res 2002;61(3):458-465. [PubMed: 12115471]

7. Krasko MY, Golenser J, Nyska A, Nyska M, Brin YS, Domb AJ. Gentamicin extended release from an injectable polymeric implant. J Control Release 2007;117(1):90-96. [PubMed: 17150275]

8. Lucke M, Schmidmaier G, Sadoni S, Wildemann B, Schiller R, Haas NP, et al. Gentamicin coating of metallic implants reduces implant-related osteomyelitis in rats. Bone 2003;32(5):521-531. [PubMed: 12753868]

9. Neut D, van de Belt H, Stokroos I, van Horn JR, van der Mei HC, Busscher HJ. Biomaterialassociated infection of gentamicin-loaded PMMA beads in orthopaedic revision surgery. $\mathrm{J}$ Antimicrob Chemother 2001;47(6):885-891. [PubMed: 11389124]

10. Walenkamp GHIM, Kleijn LLA, de Leeuw M. Osteomyelitis treated with gentamicin-PMMA beads - 100 patients followed for 1-12 years. Acta Orthop Scand 1998;69(5):518-522. [PubMed: 9855236]

11. Leggat PA, Kedjarune U. Toxicity of methyl methacrylate in dentistry. Int Dent J 2003;53(3):126131. [PubMed: 12873108]

12. Lewis G, Janna S. Estimation of the optimum loading of an antibiotic powder in an acrylic bone cement - Gentamicin sulfate in SmartSet HV. Acta Orthop 2006;77(4):622-627. [PubMed: 16929440]

13. Klemm K. The use of antibiotic-containing bead chains in the treatment of chronic bone infections. Clin Microbiol Infec 2001;7(1):28-31. [PubMed: 11284941]

14. Decher G. Fuzzy nanoassemblies: Toward layered polymeric multicomposites. Science 1997;277(5330):1232-1237.

15. Thierry B, Winnik FM, Merhi Y, Silver J, Tabrizian M. Bioactive coatings of endovascular stents based on polyelectrolyte multilayers. Biomacromolecules 2003;4(6):1564-1571. [PubMed: 14606881]

16. Vautier D, Hemmerle J, Vodouhe C, Koenig G, Richert L, Picart C, et al. 3-D surface charges modulate protrusive and contractile contacts of chondrosarcoma cells. Cell Motil Cytoskel 2003;56(3):147-158.

17. Zhu YB, Gao CY, He T, Liu XY, Shen JC. Layer-by-layer assembly to modify poly(L-lactic acid) surface toward improving its cytocompatibility to human endothelial cells. Biomacromolecules 2003;4(2):446-452. [PubMed: 12625744]

18. Picart C. Polyelectrolyte multilayer films: From physico-chemical properties to the control of cellular processes. Curr Med Chem 2008;15(7):685-697. [PubMed: 18336282]

19. Chuang HF, Smith RC, Hammond PT. Polyelectrolyte multilayers for tunable release of antibiotics. Biomacromolecules 2008;9(6):1660-1668. [PubMed: 18476743]

20. Wood KC, Chuang HF, Batten RD, Lynn DM, Hammond PT. Controlling interlayer diffusion to achieve sustained, multiagent delivery from layer-by-layer thin films. P Natl Acad Sci USA 2006;103(27):10207-10212.

21. Berg MC, Zhai L, Cohen RE, Rubner MF. Controlled drug release from porous polyelectrolyte multilayers. Biomacromolecules 2006;7(1):357-364. [PubMed: 16398536] 
22. Smith RC, Riollano M, Leung A, Hammond PT. Layer-by-layer platform technology for smallmolecule delivery. Angew Chem Int Ed Engl 2009;48(47):8974-8977. [PubMed: 19847838]

23. Dierich A, Le Guen E, Messaddeq N, Stoltz JF, Netter P, Schaaf P, et al. Bone formation mediated by synergy-acting growth factors embedded in a polyelectrolyte multilayer film. Adv Mater 2007;19(5):693-697.

24. Muller S, Koenig G, Charpiot A, Debry C, Voegel JC, Lavalle P, et al. VEGF-functionalized polyelectrolyte multilayers as proangiogenic prosthetic coatings. Adv Funct Mater 2008;18(12): 1767-1775.

25. Vazquez E, Dewitt DM, Hammond PT, Lynn DM. Construction of hydrolytically-degradable thin films via layer-by-layer deposition of degradable polyelectrolytes. J Am Chem Soc 2002;124(47): 13992-13993. [PubMed: 12440887]

26. Li BY, Jiang BB, Boyce BM, Lindsey BA. Multilayer polypeptide nanoscale coatings incorporating IL-12 for the prevention of biomedical device-associated infections. Biomaterials 2009;30(13):2552-2558. [PubMed: 19215980]

27. Schultz P, Vautier D, Richert L, Jessel N, Haikel Y, Schaaf P, et al. Polyelectrolyte multilayers functionalized by a synthetic analogue of an anti-inflammatory peptide, alpha-MSH, for coating a tracheal prosthesis. Biomaterials 2005;26(15):2621-2630. [PubMed: 15585265]

28. Aviv M, Berdicevsky I, Zilberman M. Gentamicin-loaded bioresorbable films for prevention of bacterial infections associated with orthopedic implants. J Biomed Mater Res A 2007;83A(1):1019. [PubMed: 17340599]

29. Lynn DM, Langer R. Degradable poly(beta-amino esters): Synthesis, characterization, and selfassembly with plasmid DNA. J Am Chem Soc 2000;122(44):10761-10768.

30. Gillaspy AF, Hickmon SG, Skinner RA, Thomas JR, Nelson CL, Smeltzer MS. Role of the accessory gene regulator (Agr) in pathogenesis of staphylococcal osteomyelitis. Infect Immun 1995;63(9):3373-3380. [PubMed: 7642265]

31. Oyane A, Kim HM, Furuya T, Kokubo T, Miyazaki T, Nakamura T. Preparation and assessment of revised simulated body fluids. J Biomed Mater Res A 2003;65(2):188-195. [PubMed: 12734811]

32. Erdfelder E, Faul F, Buchner A. GPOWER: A general power analysis program. Behav Res Meth Ins C 1996;28(1):1-11.

33. Anglen JO. Comparison of soap and antibiotic solutions for irrigation of lower-limb openfracture wounds - A prospective, randomized study. J Bone Joint Surg Am 2005;87A(7):1415-1422. [PubMed: 15995106]

34. Wood KC, Boedicker JQ, Lynn DM, Hammond PT. Tunable drug release from hydrolytically degradable layer-by-layer thin films. Langmuir 2005;21(4):1603-1609. [PubMed: 15697314]

35. Jewell CM, Zhang J, Fredin NJ, Wolff MR, Hacker TA, Lynn DM. Release of plasmid DNA from intravascular stents coated with ultrathin multilayered polyelectrolyte films. Biomacromolecules 2006;7(9):2483-2491. [PubMed: 16961308]

36. Changez M, Koul V, Dinda AK. Efficacy of antibiotics-loaded interpenetrating network (IPNs) hydrogel based on poly(acrylic acid) and gelatin for treatment of experimental osteomyelitis: in vivo study. Biomaterials 2005;26(14):2095-2104. [PubMed: 15576184]

37. De Giglio E, Cometa S, Cioffi N, Torsi L, Sabbatini L. Analytical investigations of poly(acrylic acid) coatings electrodeposited on titanium-based implants: a versatile approach to biocompatibility enhancement. Anal Bioanal Chem 2007;389(7-8):2055-2063. [PubMed: 17516054]

38. Salomaki M, Kankare J. Influence of synthetic polyelectrolytes on the growth and properties of hyaluronan-chitosan multilayers. Biomacromolecules 2009;10(2):294-301. [PubMed: 19119871]

39. Dubas ST, Schlenoff JB. Factors controlling the growth of polyelectrolyte multilayers. Macromolecules 1999;32(24):8153-8160.

40. Lavalle P, Gergely C, Cuisinier FJG, Decher G, Schaaf P, Voegel JC, et al. Comparison of the structure of polyelectrolyte multilayer films exhibiting a linear and an exponential growth regime: An in situ atomic force microscopy study. Macromolecules 2002;35(11):4458-4465.

41. Picart C, Mutterer J, Richert L, Luo Y, Prestwich GD, Schaaf P, et al. Molecular basis for the explanation of the exponential growth of polyelectrolyte multilayers. P Natl Acad Sci USA 2002;99(20):12531-12535. 
42. Porcel C, Lavalle P, Ball V, Decher G, Senger B, Voegel JC, et al. From exponential to linear growth in polyelectrolyte multilayers. Langmuir 2006;22(9):4376-4383. [PubMed: 16618190]

43. Porcel C, Lavalle P, Decher G, Senger B, Voegel JC, Schaaf P. Influence of the polyelectrolyte molecular weight on exponentially growing multilayer films in the linear regime. Langmuir 2007;23(4):1898-1904. [PubMed: 17279672]

44. Schlenoff JB, Dubas ST. Mechanism of polyelectrolyte multilayer growth: Charge overcompensation and distribution. Macromolecules 2001;34(3):592-598.

45. Choi J, Rubner MF. Influence of the degree of ionization on weak polyelectrolyte multilayer assembly. Macromolecules 2005;38(1):116-124.

46. Josepovitz C, Pastorizamunoz E, Timmerman D, Scott M, Feldman S, Kaloyanides GJ. Inhibition of gentamicin uptake in rat renal cortex in vivo by aminoglycosides and organic polycations. $\mathrm{J}$ Pharmacol Exp Ther 1982;223(2):314-321. [PubMed: 7131288]

47. Singh MP, Stefko J, Lumpkin JA, Rosenblatt J. The Effect of Electrostatic Charge Interactions on Release Rates of Gentamicin from Collagen Matrices. Pharm Res 1995;12(8):1205-1210. [PubMed: 7494835]

48. Stewart PS. Theoretical aspects of antibiotic diffusion into microbial biofilms. Antimicrob Agents Chemother 1996;40(11):2517-2522. [PubMed: 8913456]

49. Darouiche RO, Mansouri MD, Zakarevicz D, AlSharif A, Landon GC. In vivo efficacy of antimicrobial-coated devices. J Bone Joint Surg Am 2007;89A(4):792-797. [PubMed: 17403802]

50. Isefuku S, Joyner CJ, Simpson AHRW. Gentamicin may have an adverse effect on osteogenesis. J of Orthop Trauma 2003;17(3):212-216. [PubMed: 12621263]

51. An YH, Kang QK, Arciola CR. Animal models of osteomyelitis. Int J Artif Organs 2006;29(4): 407-420. [PubMed: 16705610]

52. Cavanaugh DL, Berry J, Yarboro SR, Dahners LE. Better prophylaxis against surgical site infection with local as well as systemic antibiotics. An in vivo study. J Bone Joint Surg Am 2009;91(8):1907-1912. [PubMed: 19651948] 


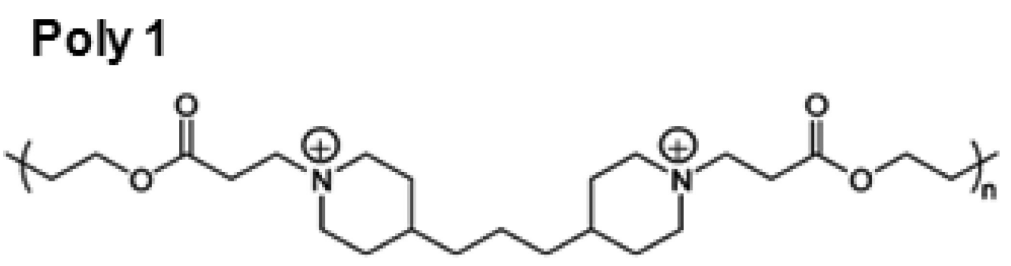

Fig. 1.

Structure of the repeat unit for the hydrolytically degradable Poly 1 cation species. 

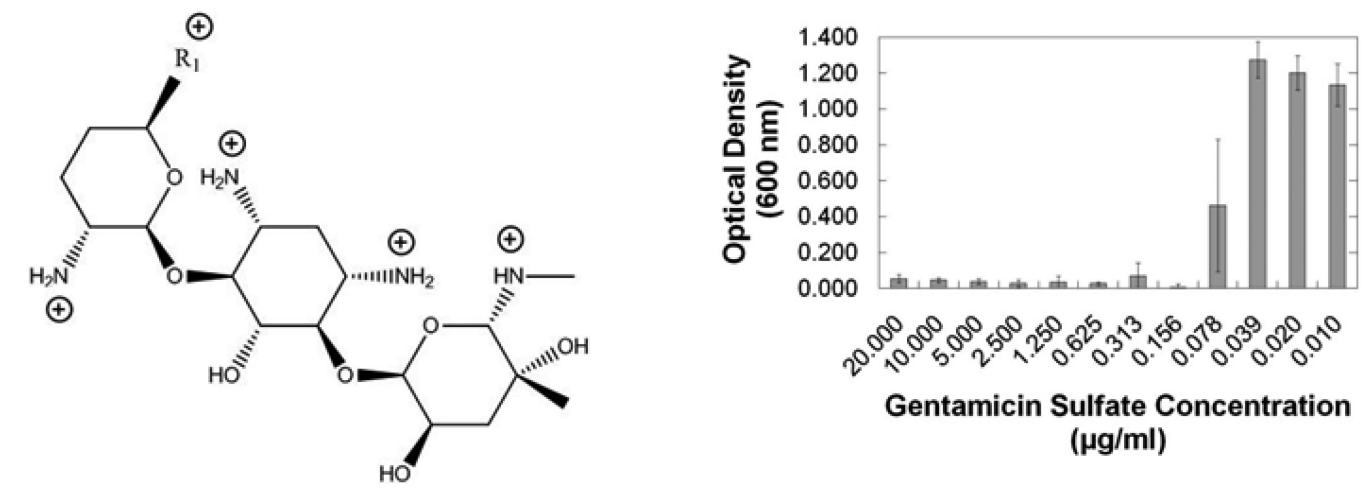

Fig. 2.

(Left) Structure of gentamicin. Sites protonated in fully charged state are indicated. Information on $\mathrm{R}_{1}$ groups and their relative content percentages can be obtained from Mediatech, Inc. (Lot \#: 61098046). (Right) In vitro efficacy of gentamicin sulfate against $S$. aureus (ATCC 49230) in CMHB over a $16 \mathrm{~h}$ incubation period at $37^{\circ} \mathrm{C}$. 


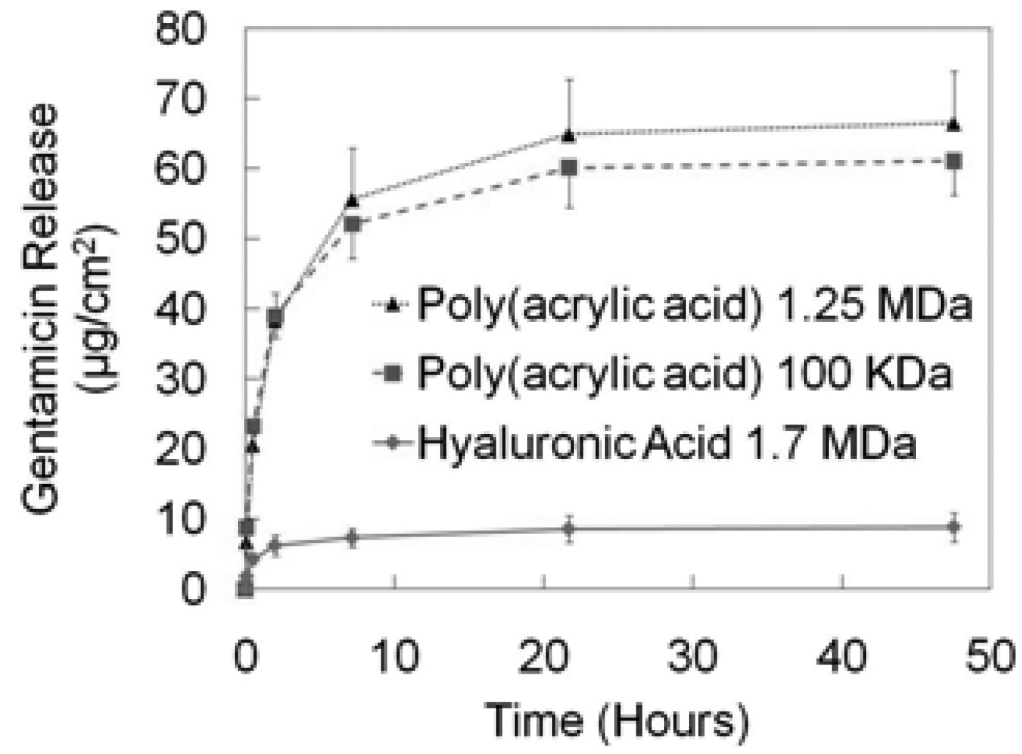

Fig. 3.

Cumulative amount of gentamicin released from [Poly 1 / Anion / GS / Anion $]_{50}$ films. 

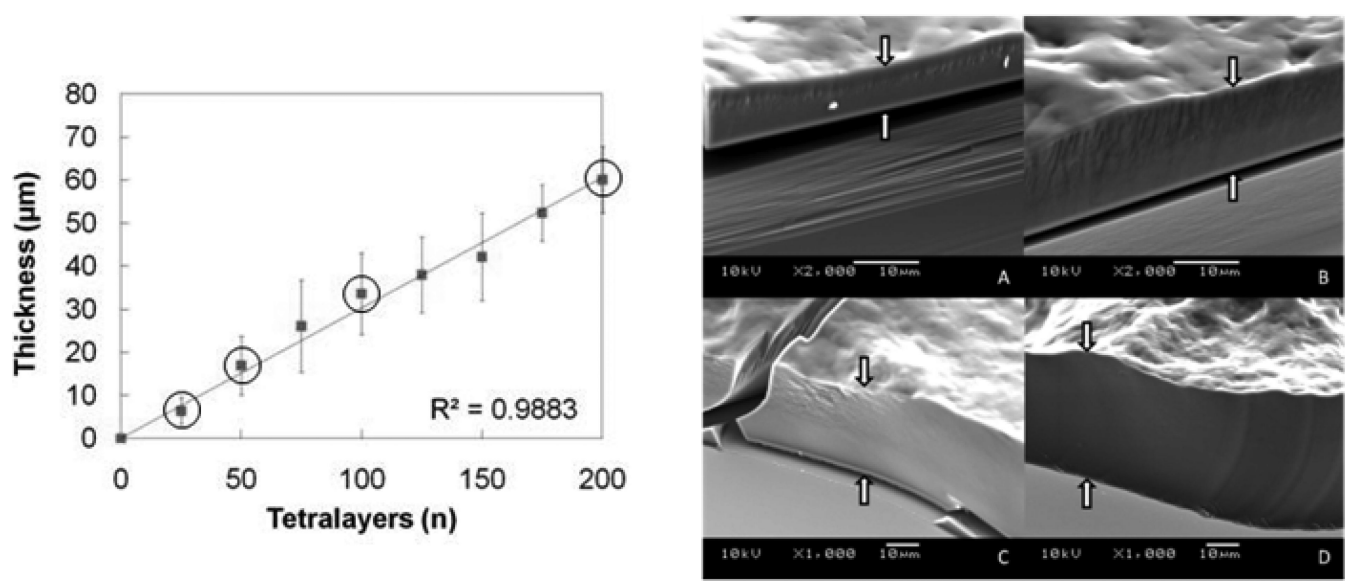

Fig. 4.

(Left) Growth curve for [Poly 1/PAA/GS/PAA $]_{n}$. SEM images are provided for the circled data points. Thicknesses were measured by profilometry at four predetermined points on each substrate and averaged over three replications. The error bars represent the average root mean squared roughness from triplicate samples and quadruplicate measurements per sample. (Right) SEM images of the growing film on a silicon surface at $(A) n=25,(B) n=$ 50, (C) $n=100$, and (D) $n=200$ layers. White arrows mark the film edge. Nota bene: the scale changes between $(\mathrm{B})$ and $(\mathrm{C})$. 

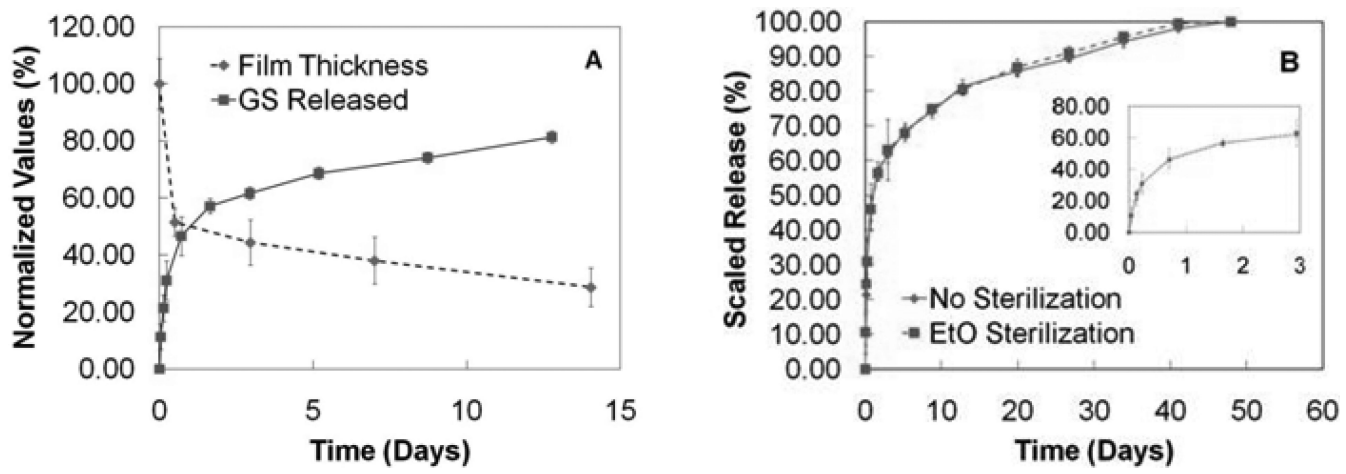

Fig. 5.

(A) Normalized film erosion from [Poly 1 / PAA / GS / PAA $]_{200}$ films and normalized GS release from [Poly $1 /$ PAA / GS / PAA $]_{200}+[\text { Poly } 1 / \text { PAA / GS }]_{1}$ films. Erosion values are normalized to initial film thickness with the error bars representing the normalized rms roughness. Release values are normalized to the final release quantity $\left(582 \mu \mathrm{g} / \mathrm{cm}^{2}\right)$. Release data have truncated for convenience of comparison. (B) Cumulative antibiotic release from $[\text { Poly 1/PAA/GS/PAA }]_{200}+[\text { Poly 1/PAA/GS }]_{1}$ films in m-SBF at $37{ }^{\circ} \mathrm{C}$ before (replotted from (A)) and after an effective dose of ethylene oxide gas. Inset contains data from the first three days. 


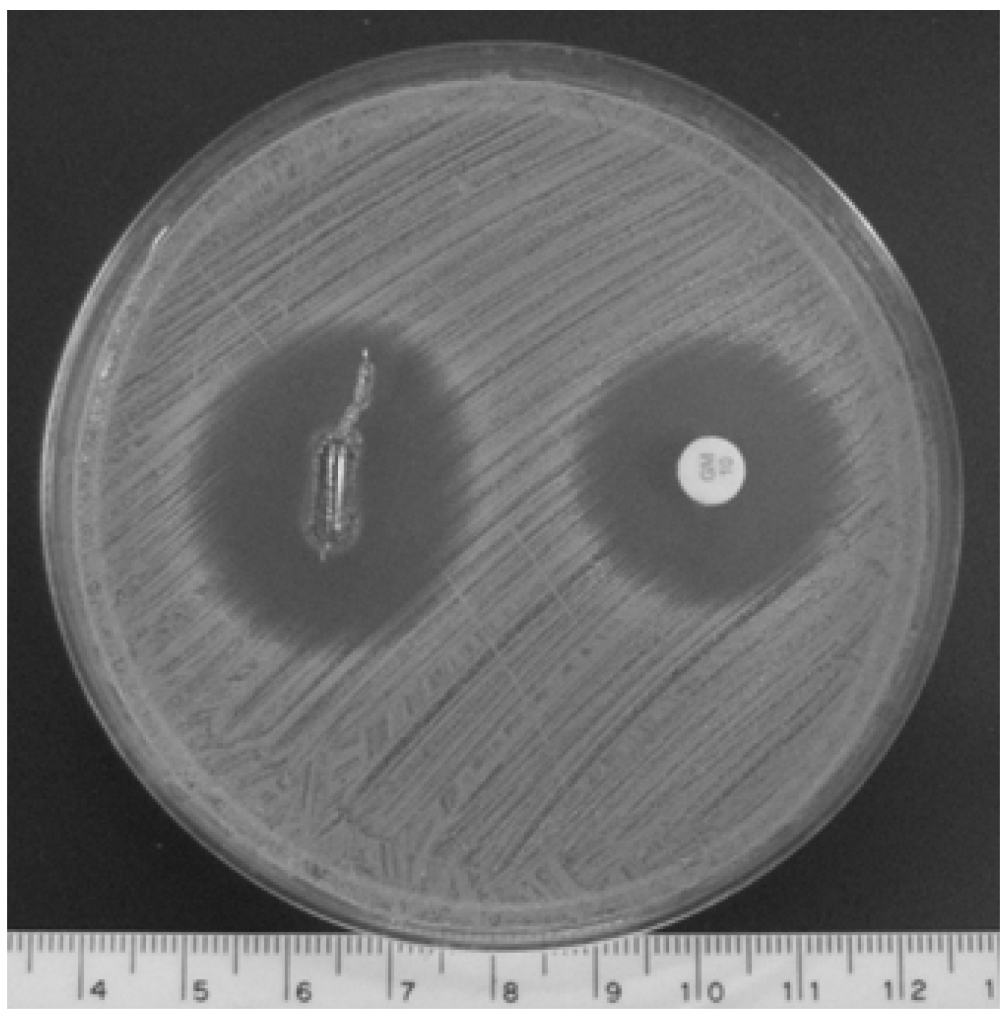

Fig. 6.

The titanium rods coated with $[\text { Poly } 1 / \mathrm{PAA} / \mathrm{GS} / \mathrm{PAA}]_{200}+[\text { Poly } 1 / \mathrm{PAA} / \mathrm{GS}]_{1}$ produced a baseline zone of inhibition of $25.6 \mathrm{~mm}$ (measured perpendicular to the long axis of the rod) against $S$. aureus after overnight incubation at $37{ }^{\circ} \mathrm{C}$. As a control, the sample is referenced to a commercially available BD Sensi-Disc. The lighter color at the rod surface is a result of the ruptured agar and not the presence of bacteria. The scale bar is in centimeters. 

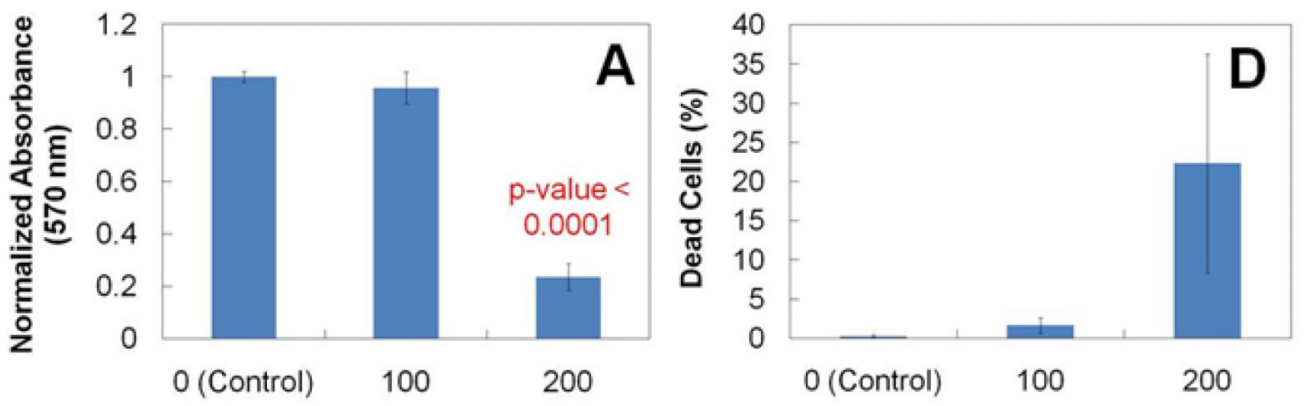

Tetralayers Eluted into Standard Medium for 1 Week
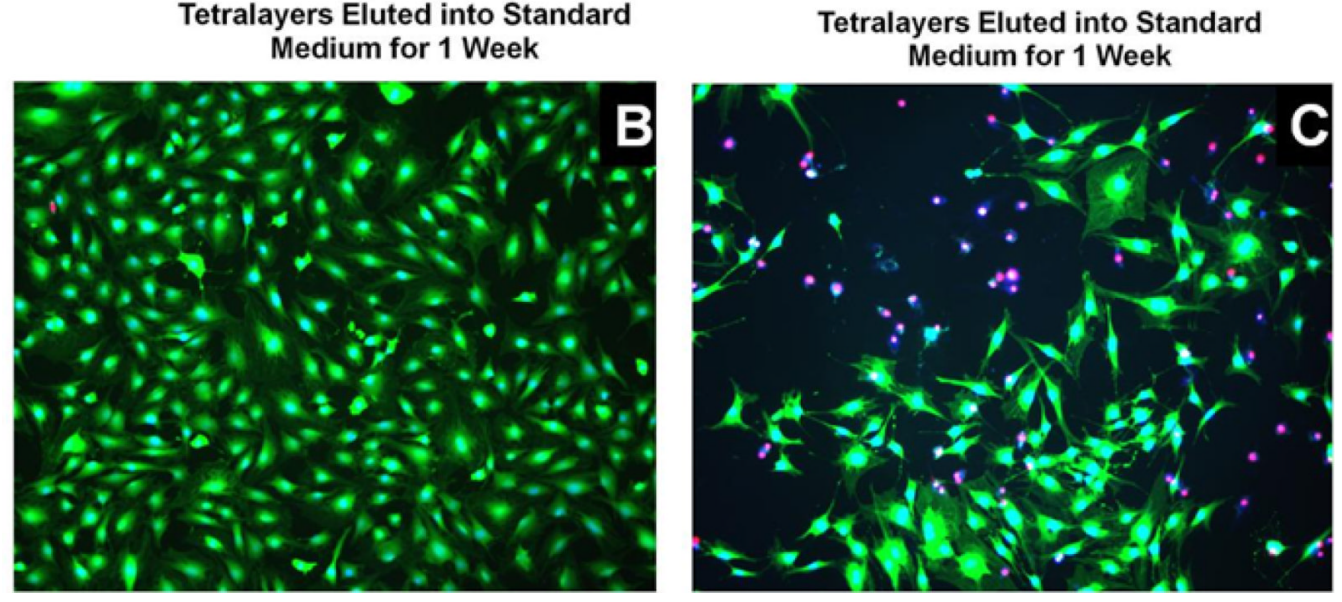

Fig. 7.

(A) MTT metabolic activity of MC3T3 cells after 16-18 hr treatment in elution buffer normalized to negative control (i.e. cells incubated in standard medium). 100x images of MC3T3 cells subjected to 16-18 hr treatment in (B) 100 and (C) 200 tetralayer elution buffer. Live cells are represented by a blue nucleus surrounded by green cytoplasm. Dead cells are represented by a red nucleus. (D) Percentage of dead cells calculated from triplicate images. 

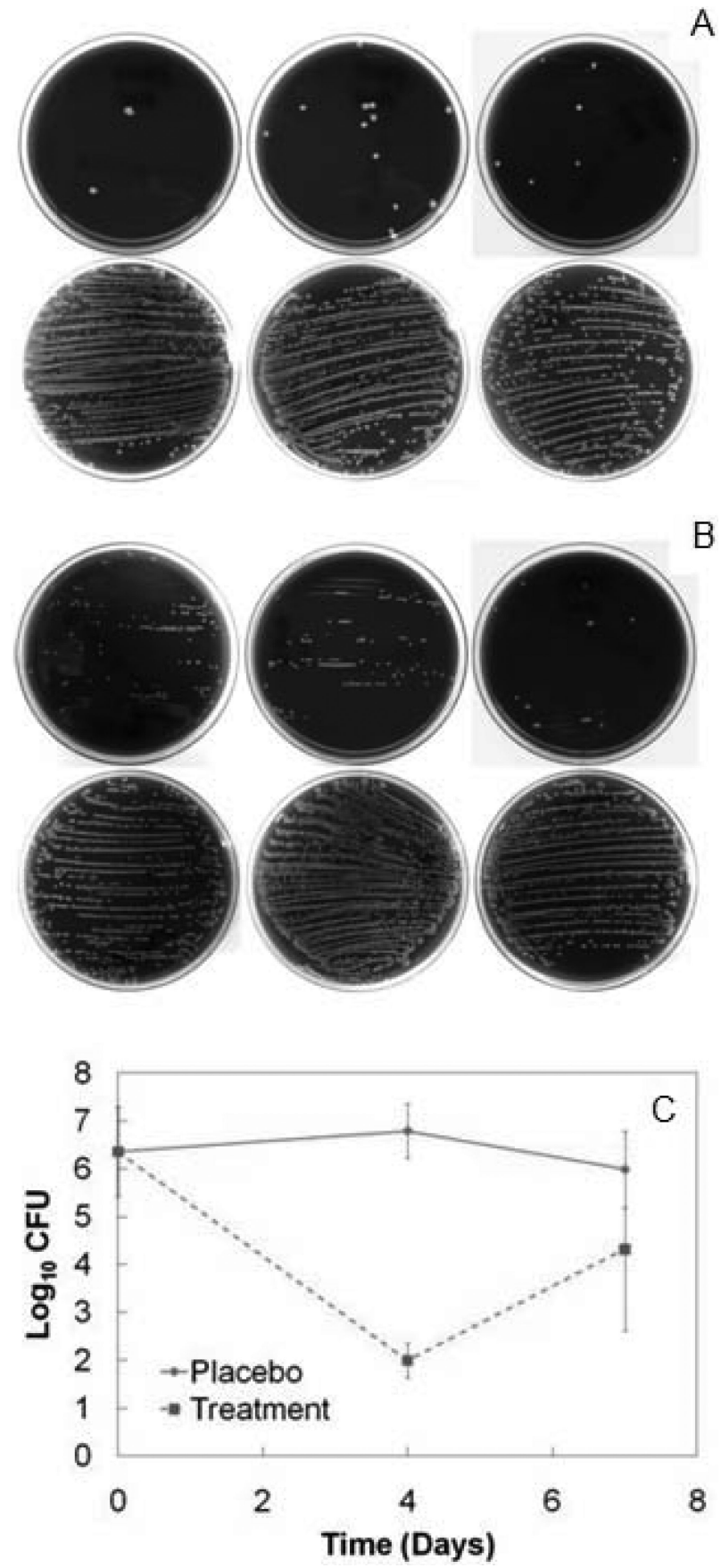

Fig. 8.

(A) Blood agar plates of explanted titanium rods that were rolled down the center of the plate and streaked for qualitative analysis of device surface colonization. Each plate corresponds to one rabbit. Each white dot corresponds to a single CFU. Explants from the four day treatment group (top row) are compared to explants from the four day placebo group (bottom row). None of the sterile plates are depicted. (B) Explants from the seven day treatment group (top row) are compared to explants from the seven day placebo group (bottom row). (C) Final counts (Mean \pm SD) of log-transformed Staphylococcus aureus CFU data in femoral condyles at day zero and after direct exchange. Raw data are available in Supplementary Table 2. 